\title{
Optical Pulse Coding Applied to Distributed Temperature Sensor Using Coherent Detection of Spontaneous Brillouin Frequency Shift
}

\author{
M. A. Soto, G. Bolognini, F. Di Pasquale
}

Scuola Superiore Sant' Anna, via G. Moruzzi 1, 56124, Pisa, Italy; email: gabriele.bolognini@sssup.it

\begin{abstract}
A 53-km Simplex-coded distributed temperature sensor based on coherent detection of Brillouin frequency shift is demonstrated with $1.1 \mathrm{~K}$ and $35 \mathrm{~m}$ resolutions, using only $10 \mathrm{~mW}$ input peak power.
\end{abstract}

\begin{abstract}
Introduction
Distributed strain and temperature sensors employing optical fibres are recently attracting a great deal of attention for industrial applications, environmental and structural monitoring [1]. While Raman scattering effect is sensitive to temperature only and is successfully exploited in distributed temperature sensing with a high accuracy and meter-scale spatial resolutions [2], Brillouin scattering in principle allows for simultaneous temperature and strain over the same optical fibre [3]. In the latter case, however, in order to distinguish temperature from strain, it is necessary to adopt suitable solutions; most commonly, Brillouin intensity and frequency shift along the sensing fibre are measured concurrently in order to obtain reliable temperature and strain profiles.
\end{abstract}

We have recently presented a Brillouin-based distributed temperature sensor (BDTS) using LandauPlaczek ratio (LPR) scheme [4], where Simplexcoding [5] was applied for SNR enhancement in directdetection of spontaneous Brillouin intensity. In this paper for first time we apply pulse coding to BDTS based on Brillouin frequency shift measurements with coherent detection [3,6]. The observed SNR improvement provided by 127-bit Simplex coding, combined with the enhanced sensitivity of the coherent receiver, allows for temperature sensing with a temperature accuracy of $1.1 \mathrm{~K}$ and a spatial resolution of $35 \mathrm{~m}$ over $53 \mathrm{~km}$ of dispersion shifted fibre (DSF), using only $10 \mathrm{~mW}$ input peak power at $1555 \mathrm{~nm}$, hence avoiding optical pulse amplification. Therefore, employing pulse coding in Brillouin sensors based on intensity and frequency shift measurements appears promising for achieving simultaneous strain and temperature sensing over the same optical fibre.

\section{Theory}

In this work we use a coherent detection scheme for measuring the Brillouin frequency shift. In such a scheme, Brillouin signal is mixed with an optical local oscillator (OLO), which allows for an amplification of the weak Brillouin light, providing at the same time efficient electric filtering of the Brillouin component [3], and bringing the further advantage of an improved dynamic range in comparison with direct detection receivers (due to square root dependence of the photocurrent on Brillouin power). It has been demonstrated that optical pulse coding provides an enhanced SNR in Brillouin intensity measurements [4], allowing for a performance improvement in LPR-based BDTS. Thus optical coding is expected to provide enhanced resolution also in Brillouin frequency measurements, since the $r m s$ noise $\delta v_{B}$ in Brillouin central frequency depends on the SNR according to:

$$
\delta v_{B}=\frac{\Delta v_{B}}{\sqrt{2}(S N R)^{1 / 4}}
$$

where $\Delta v_{B}$ is the Brillouin light linewidth [6].

In case of Simplex coding with $L$ bits, the SNRenhancement is given by the coding gain $G_{C O D}=(L+1) /(2 \sqrt{ } L)$ [5], allowing better temperature resolution. Coherent detection can in principle be used to measure both Brillouin intensity and frequency shift for simultaneous temperature and strain sensing. Hence, although the experiments reported here are aimed at temperature sensing, the results on coding benefit for Brillouin frequency shift are valid and easily applicable also to strain sensing.

\section{Experimental set-up}

In the experimental set-up, an external cavity laser $(E C L)$ at $1555.0 \mathrm{~nm}$ is used as optical source, as shown Fig. 1. In BDTS employing spontaneous scattering, the receiver is located at the same fibreend as the transmitter, so the Brillouin-OLO beat frequency $(\sim 11 \mathrm{GHz})$ can be easily detected in a conventional heterodyne receiver, since the same source is used both for pulse transmission and OLO. Thus, a part of the ECL power was split and used as OLO at receiver branch. At the transmitter-side, the ECL light is amplified by an EDFA and filtered by an optical bandpass filter (OBPF $0.5 \mathrm{~nm}$ ) for reduction of

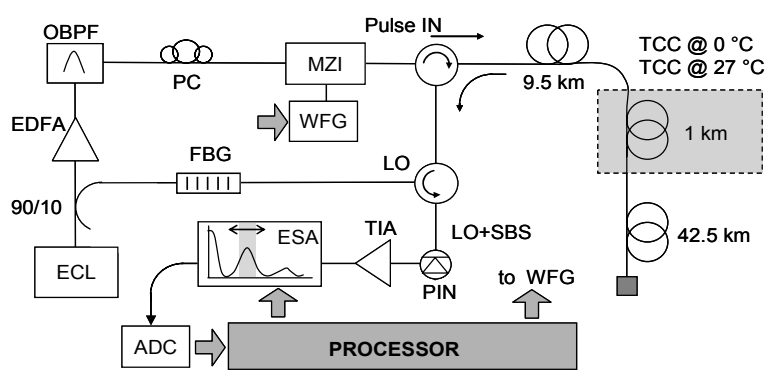

Fig. 1. Experimental set-up of coherent-detection Brillouin-based sensor 
amplified spontaneous emission (ASE). The light is then intensity-modulated through a Mach-Zehnder interferometer (MZI) in order to achieve single or 127bit Simplex-coded pulses (10 $\mathrm{mW}$ peak power). The single pulse duration is $320 \mathrm{~ns}$, corresponding to an attainable spatial resolution of $\sim 30-35 \mathrm{~m}$. The pulsed light is then coupled into the sensing fibre $(53 \mathrm{~km}$ of DSF) through an optical circulator. A length of $1 \mathrm{~km}$ of DSF is placed in a temperature controlled chamber (TCC) in order to study the sensor performance. At the receiver-side, a fibre-Bragg grating (FBG, $6 \mathrm{GHz}$ bandwidth) centred at Anti-Stokes Brillouin wavelength is used to filter-out Rayleigh and Raman scattering components. The Brillouin component is mixed with the OLO through the FBG and an optical circulator, and then detected by a $12.5 \mathrm{GHz}$ PIN photodiode followed by a trans-impedance amplifier (TIA). The OLO-Brillouin beat spectrum is acquired using a $40 \mathrm{GHz}$ electric spectrum analyser (ESA) operating in zero-span mode. To measure the Brillouin scattering spectrum accurately, OTDR traces are obtained for different beat frequencies within the Brillouin band. They are selected by electric filtering using the maximum resolution bandwidth in the ESA (3 $\mathrm{MHz}$ ), followed by averaging and data processing.

\section{Results}

Brillouin frequency shift estimation was performed by trace acquisition ( $2^{13}$ time averages) at 40 consecutive frequency intervals (starting at 10.74 $\mathrm{GHz}$ ) in $3 \mathrm{MHz}$ steps. The central frequency for each $z$ value was then reconstructed by fitting the related spectrum with a Lorentzian curve and using a least squares estimation algorithm.

Fig. 2 shows the Brillouin spectra obtained along the fibre (using Simplex coding) when TCC is set to $273 \mathrm{~K}$. The inset in Fig 2 shows a Brillouin frequency shift of $\sim 30 \mathrm{MHz}$ observed at $10 \mathrm{~km}$ distance when the TCC is set at $300 \mathrm{~K}$. The Brillouin central frequency was evaluated versus fibre distance with and without pulse coding, obtaining the temperature profiles. Fig. 3. reports the temperature distributions versus $z$ using 127-bit Simplex-coding and conventional BDTS. The experimental SNR

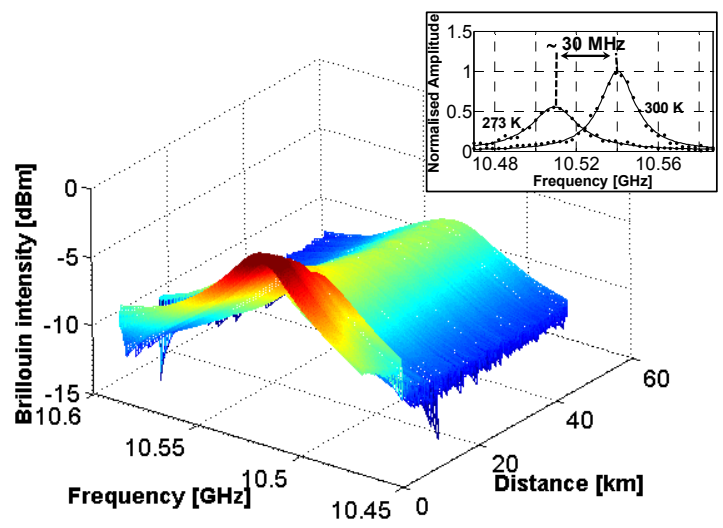

Fig. 2. 3D trace showing Brillouin scattering spectrum versus distance (inset: TCC at different temperatures)

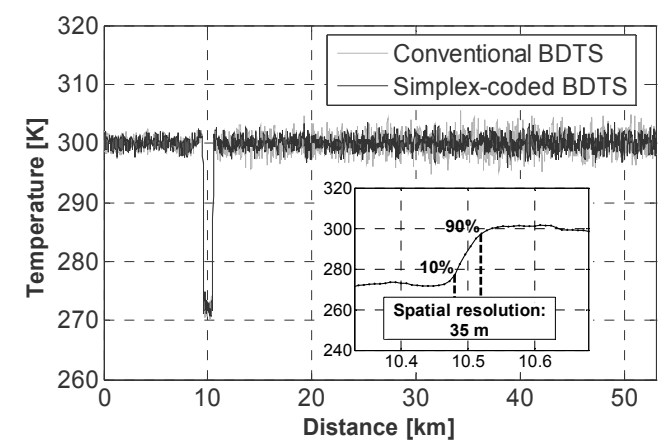

Fig. 3. Temperature distribution versus fibre distance with single pulses and Simplex-coded pulses.

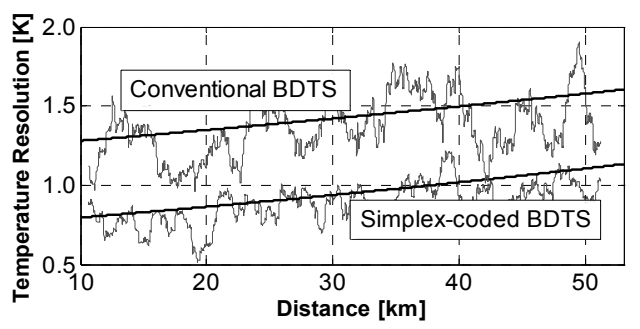

Fig. 4. Temperature resolution versus fibre distance with single pulses and Simplex-coded pulses.

enhancement provided by coding results to be 7.47 $\mathrm{dB}$, being in agreement with the theory mentioned above, predicting $7.54 \mathrm{~dB}$. The inset in Fig. 3 shows the achieved spatial resolution of $35 \mathrm{~m}$, measured as the $10 \%$ to $90 \%$ response distance. The SNR enhancement observed in our experiment allows for an average temperature resolution improvement of $\sim 1.9 \mathrm{~dB}$ compared to conventional-BDTS. This result agrees with the theoretical improvement given by the factor $(S N R)^{1 / 4}$ in Eq. (1). The temperature resolution of the BDTS is shown in Fig. 4, where the resolution of $1.6 \mathrm{~K}$ for conventional BDTS at $53 \mathrm{~km}$ distance is improved to $1.1 \mathrm{~K}$ when using 127-bit Simplex coding. In conclusion, we have shown experimentally a temperature resolution enhancement (or equivalently an increase in sensing distance) by using Simplex coding in BDTS based on Brillouin frequency shift measurement. This technique can then be successfully applied to provide performance enhancement on both strain and temperature sensing based on simultaneous Brillouin frequency shift and intensity measurements, avoiding the use of additional optical amplification.

\section{References}

1. Nature photonics, vol. 2 (2008), pp. 143-158.

2. J. Park et al, IEEE PTL, vol. 18 (2006), p. 1879-81.

3. S. M. Maughan et al, Meas. Sci. Technol., vol. 12 (2001), pp. 834-842.

4. M. A. Soto et al, IEEE Sensors J., vol. 8 (2008), pp. 225-226.

5. M. D. Jones, IEEE PTL, vol. 15 (1993), p. 822-824

6. T. Horiguchi et al, JLT, vol. 13 (1995), p. 1296-1301 